\title{
Smart Microscopy: Automation of CLEM using In situ Fluorescence Detection
}

\author{
Lucy M. Collinson ${ }^{1 *}$
}

${ }^{1}$ Electron Microscopy Science Technology Platform, Francis Crick Institute, London, UK.

* Corresponding author: lucy.collinson@crick.ac.uk

Correlative light and electron microscopy (CLEM) combines the benefits of fluorescence and electron imaging, revealing protein localisation against the backdrop of cellular architecture. The correlative imaging field is expanding rapidly, and encompasses workflows that link many different imaging modalities, to answer scientific questions in the biological and physical sciences. We link fluorescence microscopes (widefield, confocal, super-resolution and light-sheet) with electron microscopes (scanning, transmission, serial block face and focused ion beam) [1] and X-ray microscopes (microCT and soft Xray) to analyse a range of biological samples, from single cells to whole model organisms.

Our technology development work has focused on improving the speed, accuracy and accessibility of CLEM. During this development work, it became clear that the technical challenges associated with correlative imaging are exaggerated when working in 3D. To increase protein localisation precision, we developed an 'In-Resin Fluorescence' (IRF) protocol [2] that preserves the activity of GFP and related fluorophores in resin-embedded cells and tissues. The sample preparation is relatively fast, and also introduces electron contrast so that cell structure can be visualised in the electron microscope. Once the resin blocks have been cut into ultrathin sections, out-of-plane fluorescence is removed resulting in physical 'super-resolution' light microscopy in the axial direction, which increases the accuracy of the LM-EM overlays. Localisation precision is further increased by imaging the IRF sections in vacuo in the next generation of commercial integrated light and electron microscopes (ILEM). We were able to further improve accuracy by developing integrated super-resolution light and electron microscopy, using the remarkable blinking properties of GFP and YFP in-resin in vacuo [3].

With the advent of dual contrast samples comes the potential to locate and track fluorescent cells during sample preparation and automated 3D EM image acquisition. We designed and built two new locator tools - a fluorescence microscope designed to integrate with an ultramicrotome to locate cells during trimming and sectioning (the ultraLM), and an even smaller version that fits into the extremely tight space of the SBF SEM vacuum chamber for on-the-fly tracking of fluorescent cells during long automated imaging runs (the miniLM) $[4,5]$.

\section{References:}

[1] CJ Peddie and LM Collinson, Micron. 61 (2014), p. 9.

[2] CJ Peddie et al., Ultramicroscopy 143 (2014), p. 3.

[3] CJ Peddie et al., (2017) J. Struct. Biol. 199 (2017), p. 120.

[4] E Brama et al., Wellcome Open Res. 1 (2016), p. 26.

[5] This work was supported by The Francis Crick Institute which receives its core funding from Cancer Research UK (FC0010004), the UK Medical Research Council (FC0010004), and the Wellcome Trust (FC0010004); and from the UK Medical Research Council, BBSRC and EPSRC under grant award $\mathrm{MR} / \mathrm{K} 01580 \mathrm{X} / 1$. 


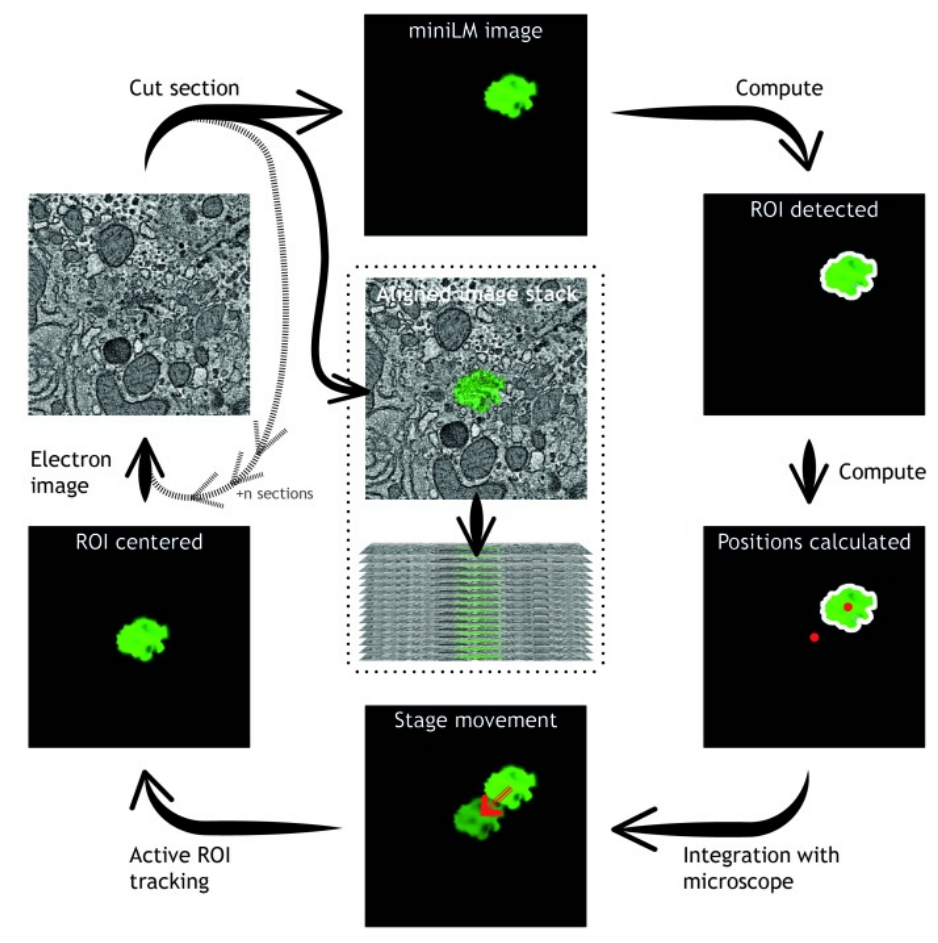

Figure 1. Automation of integrated 3D light and electron microscopy using the miniLM. Advances in automated algorithms that detect fluorescent cells in miniLM images will enable smart tracking of regions of interest during an SBF SEM data acquisition. From Brama et al. [4]. 